\title{
PLANNING THE DIESEL SUPPLY FOR OFFSHORE PLATFORMS BY A MATHEMATICAL MODEL BASED ON THE VEHICLE ROUTING PROBLEM WITH REPLENISHMENT
}

\author{
Henrique Fiorot Astoures ${ }^{\mathrm{a}}$, Rodrigo de Alvarenga Rosa $^{\mathrm{b}, *}$ André Luís da Silva Rosa ${ }^{\mathrm{c}}$ \\ a Production Engineer, Federal University of Espírito Santo, Brazil \\ ${ }^{\mathrm{b}}$ Professor, Federal University of Espírito Santo, Brazil \\ ${ }^{\mathrm{c}}$ Naval Architecture \& Marine Engineer, Federal University of Espírito Santo, Brazil
}

\begin{abstract}
Oil exploration in Brazil is mainly held by offshore platforms which require the supply of several products, including diesel to maintain its engines. One strategy to supply diesel to the platforms is to keep a vessel filled with diesel nearby the exploration basin. An empty boat leaves the port and goes directly to this vessel, then it is loaded with diesel. After that, it makes a trip to supply the platforms and when the boat is empty, it returns to the vessel to be reloaded with more diesel going to another trip. Based on this description, this paper proposes a mathematical model based on the Vehicle Routing Problem with Intermediate Replenishment Facilities (VRPIRF) to solve the problem. The purpose of the model is to plan the routes for the boats to meet the diesel requests of the platform. Given the fact that in the literature, papers about the VRPIRF are scarce and papers about the VRPIRF applied to offshore platforms were not found in the published papers, this paper is important to contribute with the evolution of this class of problem, bringing also a solution for a real application that is very important for the oil and gas business. The mathematical model was tested using the CPLEX 12.6. In order to assess the mathematical model, tests were done with data from the major Brazilian oil and gas company and several strategies were tested.
\end{abstract}




\section{INTRODUCTION}

Oil and gas are the main energy source in the world, having a strong impact on various economic sectors, especially transport and petrochemicals. Worldwide exploitation of oil happens far away from the coast at offshore platforms. These offshore platforms need to be supplied and, thus, this supply is done by fleet of boats that transports the required goods to the platforms. The pre-salt Brazilian production basins are located more than $300.0 \mathrm{~km}$ from the coast, which makes the supplying process much more complex, needing even more an accurate planning of the whole logistics to keep the platforms operating.

Diesel is very important to the platforms, without it, almost all the platforms are not able to operate. This paper deals with the diesel supply to the platforms done by a fleet of boats. Floating buoys are used to reload the boats offshore, so the boats won't have to travel all the way to the port to reload and travel back to the platforms to deliver the diesel. In the literature, the Vehicle Routing Problem with Intermediate Replenishment Facilities (VRPIRF) is the problem that is closer to the diesel supply to the platforms. Thus, this paper proposes a mathematical model, based on the VRPIRF, to plan the diesel supply to the platforms. To test the model, it was used data from the major oil company in Brazil, specifically from the Espírito Santo basin. Results showed that the use of homogeneous fleet reached the worst results and the increase on the boat's crew shift brought the best results. It also showed that the use of more buoys brings also good results.

This paper is organized as follows: In Section 2 a review with relevant works on the VRPIRF is done. Section 3 presents the proposed mathematical model. Section 4 presents the practical application and the scenarios tested. Section 5 presents the results and analysis of the results and in Section 6 the conclusions are presented.

\section{VEHICLE ROUTING PROBLEM WITH INTERMEDIATE REPLENISHMENT}

In the VRPIRF it is considered the possibility that a vehicle may make an intermediate stop at a facility during its route in order to get more load. The vehicle goes to a facility to reload its cargo compartment to keep delivering to the clients. This is important, especially when the depot is far from the client zone, avoiding the vehicles to travel back to the depot and return to the client zone.

Tarantilis et al. (2008) used three metaheuristics, Variable Neighborhood Search (VNS), Tabu Search (TS) and Guided Local Search (GLS) to solve the VRPIRF. They did the tests with benchmark instances. Schneider et al. (2014a) proposed the Electric Vehicle Routing Problem with Time Windows (E-VRPTW), similar to VRPIRF. They proposed a mathematical model and a hybrid heuristic that combines VNS and TS for the intensification phase of the VNS. They tested their problem with benchmark instances. Schneider et al. (2014b) proposed the Vehicle Routing Problem with Intermediate Stops (VRPIS), similar to E-VRPTW, that also deals with routes of electric vehicles considering visits to intermediate facilities in order recharge them with electric energy. They also considered visits to other intermediate facilities to reload the vehicle cargo compartment. It is also presented a metaheuristic Local Search (LS) with Adaptive Variable Neighborhood Search (AVNS) to solve the problem. They tested their problem with benchmark instances.

Angelelli e Speranza (2002) proposed the Periodic Vehicle Routing Problem with Intermediate Facilities (PVRP-IF) 
and also proposed a TS for the PVRP-IF. They tested with benchmark instances. Brandão and Pureza (2015) proposed a Mixed Integer Linear Programming (MILP) mathematical model, based on the E-VRP, applied to a dairy company, where the cargo has to have its temperature cooled by truck connection to electrical outlets. Ghiani et al. (2001) proposed the Capacitated Arc Routing with Intermediate Facilities (CARPIF) which is similar to the VRPRF. The difference is that VRPRF considers routing in the nodes and CARPIF considers routing in arcs. They tested on benchmark instances. Polacek et al. (2008) proposed a VNS to solve the CARPIF.

After this review, it can be seen that few papers were published about the VRPIRF, and none of them dealt with a practical application. Specifically, for the oil and gas logistics no paper was published yet. So, this paper brings a new application to the VRPRF, the problem of supplying diesel to offshore platforms, and a model adapted with some specific constraints for the problem.

\section{PROPOSED MATHEMATICAL MODEL}

The proposed VRPRF mathematical model applied to the supply of diesel to offshore platforms considers that each boat starts its trip at the port completely empty of cargo. Then it goes strictly to a buoy, that is called in the model as facility, to load cargo and after that it will start delivering to the platforms. After the boat holds are empty, it goes to one facility to reload and then starts a new trip to deliver to the platforms. This process is repeated until the boat's crew shift is finished. At this time, the boat must return to the port, called in the model as virtual port, to finish its route. Thus, it is defined that $n$ is the number of platforms to be served, $n f$ is the number of available facilities, i.e., buoy, and $n b$ the number of available boats.

With these definitions, the sets used by the model can be defined as $V$ is the set of nodes that ranges from 1 to $n p+$ $n f ; V 0$ is an auxiliary set which adds the port to set $V$ that ranges from 0 to $n p+n f ; V 1$ is an auxiliary set which adds the virtual port to set $V$, that ranges from 1 to $n p+n f+1 ; V T$ is an auxiliary set which adds the initial and virtual port, that ranges from 0 to $n p+n f+1 ; F$ is the set of facilities that ranges from 1 to $n f$, i.e., to the model it was defined a maximum limit $v f$ that a boat can visit one facility, so, each facility in the set $F$ occurs $v f$ times; $F 0$ is an auxiliary set of facilities which adds the port to set $F$ that ranges from 0 to $n f ; P$ is the set of platforms that ranges from 1 to $n p ; P 0$ is an auxiliary set of platforms which adds the port to set $P$ that ranges from 0 to $n p$; and $B$ is the set of boats that ranges from 1 to $n b$.

The parameters of the model are: $d_{i j}$ is the distance between nodes $i, j \in V T ; t_{i j}$ is the traveling time between node $i, j \in V T ; q_{b}$ is the boat's $b \in B$ load capacity in tons of diesel,; $c b_{b}$ is the cost to use boat $b \in b ; c k_{b}$ is the cost of each kilometer traveled by boat $b \in B ; u_{p}$ is the total demand in tons of platform $p \in P 0, u_{0}=0 ; t s_{p}$ is the time to unload a ton (delivery) at platform $p \in P 0, t s_{0}=0 ; t d c_{p}$ is the approach maneuver time to start the operation at platform $p \in P 0, t d c_{0}=0 ; u f_{f}$ is the load capacity at facility $f \in F 0, u f_{0}=0 ; t d f_{f}$ is the approach maneuver time to start the operation at facility $f \in F ; t c_{f}$ is the time to load a ton into a boat at facility $f \in F$; $t m x$ is the maximum traveling time of a boat, i.e., the boat's crew shift period; and $M$ is a logical number to the model that assumes a big value.

The decision variables of the mathematical model are: $x_{i j b}$ is a binary variable that has value equal to 1 if the boat $b \in B$ travels from node $i \in V T$ to node $j \in V T$ and 0 , otherwise; $l_{i b}$ is the total load of boat $b \in B$ when leaving 
node $i \in V T$; and $a_{i b}$ is the arrival time of boat $b \in B$ at node $i \in V T$. The objective function and the constraints of the model are presented next.

Objective Function

Minimize

$\sum_{i \in V 0} \sum_{j \in V 1 \mid i \neq j} \sum_{b \in B} d_{i, j} x_{i, j, b} c k_{b}+\sum_{b \in B} c b_{k} \sum_{j \in V} x_{0, j, b}$

Constraints

$\sum_{b \in B} \sum_{i \in V 0 \mid i \neq p} x_{i, p, b}$
$\sum_{j \in V} x_{0, j, b} \leq 1$

$\forall p \in P$

$\sum_{i \in V 0 \mid i \neq j} x_{i, j, b}-\sum_{i \in V 1 \mid i \neq j} x_{j, i, b}=0$

$\forall b \in B$

$a_{i, b} \leq t m x$

$\forall j \in V, b \in B$

$a_{p, b}+\left(\left(t_{p, j}+t d c_{p}+u_{p} t s_{p}\right) x_{p, j, b}\right)$

$\forall i \in V T, b \in B$

$-\left(M\left(1-x_{p, j, b}\right)\right) \leq a_{j, b}$

$\forall p \in P 0, j \in V 1, b \in B$

$\mid p \neq j$

$a_{f+n, b}+\left(\left(t_{f+n p, j}+t d f_{f}+q_{b} t c_{f}\right) x_{f+n p, j, k}\right)$

$\forall f \in F, j \in V 1, b \in B$

$-\left(M\left(1-x_{f+n, j, b}\right)\right) \leq a_{j, b}$

$\mid(f+n p) \neq j$

$x_{p, j, b}=0$

$\forall p \in P 0, j \in V 1, b \in B$

I $(p=0)$ and $(j<n p)$

$l_{j, b} \leq l_{p, b}-u_{p} x_{p, j, b}+M\left(1-x_{p, j, b}\right)$

$\forall p \in P 0, j \in V 1, b \in B$

I $(p \neq 0)$ and $(p \neq j)$

$l_{j, b} \geq l_{p, b}-u_{p} x_{p, j, b}-M\left(1-x_{p, j, b}\right)$

$\forall p \in P 0, j \in V 1, b \in B$

I $(p \neq 0)$ and $(p \neq j)$

$l_{j, b}=0$

$\forall f \in F 0, j \in V 1, b \in B$

$\mathrm{I}(f=0)$ and

$(j \geq(n p+1))$ and

$(j \leq(n p+n f))$

$l_{j, b} \leq l_{f+n p, b}+u_{f} x_{f+n p, j, b}+M\left(1-x_{f+n p, j, b}\right)$

$\forall f \in F 0, j \in V 1, b \in B$

$\mid(f \neq 0)$ and $((f+n p) \neq j)$

$l_{i, b} \leq q_{b}$

$\forall i \in V 1, b \in B$

$l_{n+n f+1, b}=0$

$\forall b \in B$

$l_{0, b}=0$

$\forall b \in B$

$a_{i, b} \geq 0$

$\forall i \in V T, b \in B$

$l_{i, b} \geq 0$

$\forall i \in V 1, b \in B$ 
The objective function, Equation (1), represents the total logistics costs and it is divided into two parts. The first one represents the total sailing cost of a boat to sail from one node to another node. The second part represents the sum of the costs to use each boat. The objective function must be minimized. Constraints (2) ensure that each platform is visited once. Constraints (3) states that each boat can leave the port to a platform or to a facility at most. Constraints (4) ensure that if the node $i \in V$ is visited by a boat $k \in K$, then this same boat must leave this node. It is called as the network flow constraint. Constraints (5) ensure that the arrival time at any node, including the virtual port, must be smaller or equal to the maximum traveling time of a boat. Constraints (6) guarantees that the arrival time at platform $p \in P$ plus its approach maneuver time plus its delivering time, calculated as the amount of cargo delivered multiplied by the operation time per ton, plus the traveling time from platform $p \in P$ to another node must be less than or equal to the arrival time of the boat at this node.

Constraints (7) guarantees that the arrival time at facility $f \in F$ plus its approach maneuver time plus its loading time, calculated as the amount of cargo loaded multiplied by the loading time per ton, plus the traveling time from facility $f \in F$ to another node must be less than or equal to the arrival time of the boat at this node. Constraints (8) define that a boat cannot travel from the port, $p=0$, directly to a platform $p \in P$, i.e., the boat must travel first to a facility. Constraints (9) and (10) define that if a boat travel from a platform $p \in P$ to any other node $j \in V 1$ different from itself, the total load of the boat arriving in node $j \in V 1$ is calculated as the total load at platform $p \in P$ minus the load delivered at platform $p \in P$. Constraints (11) define that the load of a boat arriving at a facility traveling from a port, $f=0$, is equal to zero. Constraints (12) define that the load of a boat arriving at node $j \in V 1$ traveling from a facility $f \in F$ is less than or equal to the total load of the boat arriving at facility $f \in F$ plus the total cargo loaded at facility $f \in F$.

Constraints (13) define the load in the boat at node must be smaller than or equal to boat load capacity. Constraints (14) guarantee that a boat when arriving to the virtual port, i.e., finishing its route must have its load equal to zero, i.e., must return empty to the port. Constraints (15) guarantee that a boat leaving the port has its load equal to zero, i.e., it starts the rout empty. Constraints (16) and (17) define, respectively that for all nodes the arriving time at the node and the total load leaving the node is equal to or greater than zero. Constraints (18) define $x_{i, j, b}$ as binary variables.

\section{STUDY CASE AND SCENARIOS DEFINITION}

To transport diesel to the platforms it is used three types of boats named Platform Supply Vessel (PSV): PSV 1500, and PSV 3000, which have holds with, respectively, 1,500ton and 3,000ton. The distance matrix between all nodes was set by calculating the Euclidean distance using the georeferenced coordinates of the port, the platforms and the buoys. The travel time matrix between all nodes was obtained by dividing the distance matrix by the average speed of the boats that was considered $18.52 \mathrm{~km} / \mathrm{h}$. The operation time of the boat to unload at the platforms depends on the amount of cargo to be delivered and it is calculated as the total cargo to be delivered multiplied by the time to unload a ton. This time to unload is dependent of the platforms gears and it was informed by the oil company for each platform. The approach maneuver time at a platform was considered the same for all platforms, an average of 1 hour and 56 minutes. The operation time of the boat to load at the buoy depends on the amount of cargo to be loaded 
and it is calculated as the total cargo to be loaded multiplied by the time to load a ton. This time is dependent of the pumps to load the diesel inside the boats holds and it was informed by the oil company for each buoy. The approach maneuver time at a buoy was considered the same for all buoys, an average of 1 hour and 50 minutes.

Group 1 represents the actual operation of the oil company that usually it has 8 operating platforms and 2 in maintenance, sometimes all of them are operating. Group 2 evaluates the impact to have more buoys, it has 3 instances like Group 1, varying only the number of buoys. Group 3 evaluates the impact of a homogenous fleet with only small and bigger boats, it has 2 instances equal to Instance 5, the one that achieved the best result. Instance 7 has only small boats and Instance 8 has only bigger boats. Group 4 tests the impact of the increase in the period of the boat's crew shift from the established 8 days to 16 days, it is based on Instance 5. Finally, Group 5 tests how will CPLEX deals with bigger instances.

Table 1 shows the instances tested. In Table 1, column Group represent the group that the instance belongs. Column Instance is the number of the instance. Column Number of Platforms represents the number of platforms considered in each instance. Column Number of buoys represents the number of buoys available to meet the demands of the platforms. The nest three columns bring information about the fleet of boats. Column Mix represents the type of the fleet: HG, for the heterogeneous fleet and HM for homogeneous fleet. Column PSV 1500 represents the total number of boats of type PSV 1500. Column PSV 3000 represents the total number of boats of type PSV 3000. Column Number of is the mix of the fleet used in each instance. The sixth column is the types of boats available for service. The seventh column represents the amount of each type of boats available in each instance. The eighth column is the boat's crew shift period, i.e., the amount of time that a boat can sail without returning to port.

\begin{tabular}{|c|c|c|c|c|c|c|c|}
\hline \multirow[b]{2}{*}{ है } & \multirow{2}{*}{ 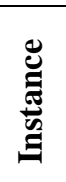 } & \multirow{2}{*}{$\begin{array}{c}\text { Number of } \\
\text { Platforms (un) }\end{array}$} & \multirow{2}{*}{$\begin{array}{l}\text { Number of } \\
\text { Buoys (un) }\end{array}$} & \multicolumn{3}{|c|}{ Fleet } & \multirow{2}{*}{$\begin{array}{l}\text { Shift Period } \\
\quad \text { (days) }\end{array}$} \\
\hline & & & & Mix & $\begin{array}{l}\text { PSV } 1500 \\
\text { (un) }\end{array}$ & $\begin{array}{l}\text { PSV } 3000 \\
\quad(\text { un })\end{array}$ & \\
\hline \multirow{3}{*}{1} & 1 & 6 & \multirow{3}{*}{1} & \multirow{3}{*}{$\mathrm{HG}$} & \multirow{3}{*}{3} & \multirow{3}{*}{3} & \multirow{3}{*}{8} \\
\hline & 2 & 8 & & & & & \\
\hline & 3 & 10 & & & & & \\
\hline \multirow{3}{*}{2} & 4 & 6 & \multirow{3}{*}{2} & \multirow{3}{*}{$\mathrm{HG}$} & \multirow{3}{*}{3} & \multirow{3}{*}{3} & \multirow{3}{*}{8} \\
\hline & 5 & 8 & & & & & \\
\hline & 6 & 10 & & & & & \\
\hline \multirow{2}{*}{3} & 7 & 8 & \multirow{2}{*}{2} & \multirow{2}{*}{$\mathrm{HM}$} & 6 & 0 & \multirow{2}{*}{8} \\
\hline & 8 & 8 & & & 0 & 6 & \\
\hline 4 & 9 & 8 & 2 & $\mathrm{HG}$ & 3 & 3 & 16 \\
\hline 5 & 10 & 15 & 2 & $\mathrm{HG}$ & 4 & 4 & 16 \\
\hline
\end{tabular}

\section{Table 1 - Test instances}

\section{RESULTS AND ANALYSIS}


It was used CPLEX 12.6 to solve the instances and it was defined a time limit of 4 hours to run each instance. It was used a computer with an Intel core $i 7$ with 16GB. In Table 2, column Group represent the group that the instance belongs. Column Instance is the number of the instance. Column OF, UB e LB represents, respectively, the value of the objective function. The upper bound and the lower bound. Column GAP shows the gap that CPLEX got after executing for 4 hours. If the gap is equal to zero, then CPLEX got the optimal solution. Column Exec. Time represents how many seconds CPLEX run to reach a solution, if it is below for hours, then gap equal to zero and the optimal solution was reached. Column Used Boats, PSV 1500 and PSV 3000 represents, respectively, how many boats was used for each type in the solution. Last Column Total Distance represents how many kilometers was sailed by all used boats.

Based on Table 2, CPLEX found the optimal solution for 6 instances in a short period of time. Even for the instances with gap, all gaps were very small for 4 hours CPLEX execution. For information safety reasons, the major Brazilian oil company did not allow us to compare the CPLEX results with its results. Thus, based on Table 2, it is presented next an analysis comparing each group representing different strategies to plan the diesel supply to the platforms.

\begin{tabular}{|c|c|c|c|c|c|c|c|c|c|}
\hline \multirow{2}{*}{ 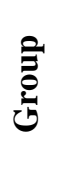 } & \multirow{2}{*}{ 导 } & \multirow{2}{*}{$\begin{array}{c}\text { OF (US\$ } \\
\times 100)\end{array}$} & \multirow{2}{*}{$\begin{array}{c}\text { UB (US\$ } \\
\text { x 100) }\end{array}$} & \multirow{2}{*}{$\begin{array}{c}\text { LB } \\
(\mathrm{US} \$ \mathbf{x} \\
\mathbf{1 0 0})\end{array}$} & \multirow{2}{*}{$\begin{array}{l}\text { GAP } \\
(\%)\end{array}$} & \multirow{2}{*}{$\begin{array}{c}\text { Exec. Time } \\
\text { (s) }\end{array}$} & \multicolumn{2}{|c|}{ Used Boat (un) } & \multirow{2}{*}{$\begin{array}{c}\text { Total } \\
\text { distance } \\
\text { (km) }\end{array}$} \\
\hline & & & & & & & $\begin{array}{l}\text { PSV } \\
1500\end{array}$ & $\begin{array}{l}\text { PSV } \\
3000\end{array}$ & \\
\hline \multirow{3}{*}{1} & 1 & 970.0 & 970.1 & 970.1 & 0.00 & 33.83 & 1 & 0 & 907.0 \\
\hline & 2 & $2,927.0$ & $2,927.0$ & $2,927.0$ & 0.00 & 3319.95 & 2 & 1 & $4,400.6$ \\
\hline & 3 & $2,924.5$ & 2924.5 & $2,923.1$ & 3.11 & 14400.61 & 3 & 0 & $4,419.3$ \\
\hline \multirow{3}{*}{2} & 4 & 969.4 & 969.4 & 969.4 & 0.00 & 254.72 & 0 & 1 & 835.9 \\
\hline & 5 & $1,958.9$ & 1958.9 & $1,958.9$ & 0.00 & 4594.53 & 1 & 1 & $3,694.8$ \\
\hline & 6 & $1,956.2$ & 1956.2 & $1,955.0$ & 3.29 & 14400.0 & 1 & 1 & $3,603.1$ \\
\hline \multirow{2}{*}{3} & 7 & $2,926.6$ & 2926.6 & $2,921.3$ & 11.30 & $14,400.0$ & 3 & 0 & $4,356.2$ \\
\hline & 8 & $2,922.4$ & 2922.4 & $2,922.4$ & 15.45 & $5,071.3$ & 0 & 2 & $3,937.8$ \\
\hline 4 & 9 & 994.8 & 994.8 & 994.8 & 0.00 & 242.11 & 0 & 1 & $3,375.7$ \\
\hline 5 & 10 & $1,963.9$ & $1,963.9$ & $1,952.3$ & 12.61 & $14,400.0$ & 0 & 2 & $3,676.6$ \\
\hline
\end{tabular}

Table 2 - CPLEX Results

It can be seen from Table 2, Group 1 and 2, that putting more buoys available to load the boats leads to a better solution. Instances 4, 5 and 6 had smaller objective function than Instances 1, 2 and 3. It also can be noted that using homogenous fleet with smaller vessel, Instance 7, or with bigger vessels, Instance 8, when compared with Instance 5 , with heterogeneous fleet, lead to worst solutions for the model, showing that probably the use of homogeneous fleet is not a good option. This happened, because only using small vessels, the boats must make more trips to be reloaded at the buoys and, so, the total distance is bigger and also the cost of a smaller boat is not so cheaper than a bigger boat, and thus, a reduction in one unit is not enough to make the total cost smaller. Instance 8 used 2 boats, but both are bigger boats, that are more expensive than one small and one big boat. When analyzing the increment of the boat's crew shift, Group 4, Instance 9, it can be noted that this strategy brings the greatest reduction in the total cost than the other strategies. Although these good results, it is important to mention that Unions agreements to 
change the boat's crew shift are very hard to be done in Brazil, and so, it is not possible to say that this strategy is eligible to be put in practice. Instance 10 represents a two times growth of the logistics done nowadays and it was proposed to test if CPLEX could found a solution. CPLEX found a solution with a gap of $12,61 \%$ that is not the optimal solution, but it shows that CPLEX can handle instances that represents problems bigger than the actual scenario and that are difficult to be implemented, because is too expensive to install and drill eight more oil wells.

After all the analyzes done, it is possible to say that the proposed mathematical model can be an important tool to help planning the diesel supply logistics and the results may help the logistics planners to analyze different strategies to propose cheaper logistics strategies keeping the logistics service level.

\section{CONCLUSION}

This paper proposed a mathematical model to the VRPIRF adapted to the diesel supply to offshore platforms with specific constraints for the problem. Almost all the previous paper has dealt with theoretical applications, this paper deals with a real application to the model. The application was in a major Brazilian oil company and several analyses were done.

From the results achieved by CPLEX, some conclusions were reached. The strategy to put more buoys to reload the boats may lead to lower costs. The strategy of using homogenous fleet, even with small or bigger vessels, did not show to be a good strategy, leading to higher logistics costs. By the end, the best strategy was to increase the boat's crew shift, besides it is very hard to be put in practice because of the Unions agreements.

It is suggested for future researches the implementation of a metaheuristic to reach solutions for instances with more platforms, boats and buoys, since the problem is NP-Hard and CPLEX is not able to find optimal solutions for bigger instances.

\section{Acknowledgements}

The authors thank FAPES (458/2013) and CNPq (477357/2013-0) for the financial support.

\section{REFERENCES}

ANGELELLI, E. and SPERANZA, M. G. (2002). The periodic vehicle routing problem with intermediate facilities. European journal of Operational research, 137(2), pp. 233-247.

BRANDÃO, R.; PUREZA, V. (2015). Distribuição de laticínios com restrições de recarga via programação matemática. XXIX Anpet, 9-13 Novembro 2015, Ouro Preto.

GHIANI, G.; IMPROTA, G.; LAPORTE, G. (2001). The capacitated arc routing problem with intermediate facilities. Networks, 37(3), pp. 134-143.

POLACEK, M.; DOERNER, K. F.; HARTL, R. F.; MANIEZZO, V. (2008). A variable neighborhood search for the capacitated arc routing problem with intermediate facilities. Journal of Heuristics, 14(5), pp. 405-423.

SCHNEIDER, M.; STENGER, A.; GOEKE, D. (2014a). The electric vehicle routing problem with time windows and recharging stations. Transportation Science, INFORMS, v. 48, n. 4, pp. 500-520.

SCHNEIDER, M.; STENGER, A.; HOF, J. (2014b). An adaptive vns algorithm for the vehicle routing problem with 
intermediate stops. OR Spectrum, Springer, pp. 1-35.

TARANTILIS, C. D.; ZACHARIADIS, E. E.; KIRANOUDIS, C. T. (2008). A hybrid guided local search for the vehicle-routing problem with intermediate replenishment facilities. Journal on Computing, INFORMS, 20(1), pp. 154-168. 